\title{
An examination of the accident and emergency management of deliberate self harm
}

\author{
Michael Dennis, Marie Beach, Phillip Adrian Evans, Anthony Winston, Trevor Friedman
}

Division of Psychiatry for the Elderly,

University of Leicester, Leicester General Hospital, Leicester M Dennis

M Beach

Accident and
Emergency
Department, Leicester
Royal Infirmary,
Leicester
P A Evans

Department of Psychiatry, University of Leicester, Leicester General Hospital,

Leicester

A Winston

Leicestershire Mental Health Service NHS

Trust, Leicester

General Hospital,

Leicester

T Friedman

Correspondence to:

Dr M S Dennis, Division of Psychiatry for the Elderly,

Leicester General Hospital,

Gwendolen Road, Leicester

LE5 4PW.

Accepted for publication 2 May 1997

\begin{abstract}
Objective-To examine the adequacy of assessment and management of deliberate self harm (DSH) undertaken by accident and emergency (A\&E) medical staff.

Methods-The records for attendances to the Leicester Royal Infirmary A\&E department with a diagnosis of "self inflicted" injury for the 12 month period April 1994 to March 1995 were scrutinised. If the episode was identified as DSH, then assessment and management were examined, using an instrument based on the Royal College of Psychiatrists' standards of service for the general hospital management of adult DSH.
\end{abstract}

Results-There were 934 episodes of DSH involving 854 patients. The mean age was 32 (SD 14.2), with an even sex distribution. Overdose was by far the most common method of DSH (91.5\%). Information concerning suicide intent was documented in $70 \%$ of cases, and psychiatric history in $67 \%$. Less information was recorded for medical history $(50 \%)$, mental state (51\%), recent stress $(55 \%)$, or previous DSH (47\%), and only $23 \%$ had an assessment of risk of further DSH. Very little was recorded concerning alcohol or substance misuse. In 291 cases (31\%), the patient was discharged directly home by A\&E medical staff, and 50 of these were referred for psychiatric outpatient follow up; $210(23 \%)$ were referred for specialist assessment in the department and 423 $(45.5 \%)$ were admitted to medical/surgical wards. The frequency with which information was recorded varied significantly between outcome groups. At night A\&E staff were far more likely to discharge a patient home themselves than refer for specialist assessment $(P<00.001)$.

Conclusions-With over half the sample not admitted, the responsibility for the initial risk assessment lies with A\&E medical staff. The study reveals a need for improved planning and delivery of services.

( $尹$ Accid Emerg Med 1997;14:311-315)

Keywords: deliberate self harm; suicide risk; accident and emergency department

Deliberate self harm (DSH) is a frequent cause of acute medical admission, ${ }^{1}$ but many patients are discharged directly from accident and emergency $(\mathrm{A} \& \mathrm{E}) .^{2}$ Approximately $1 \%$ of people who attend hospital after DSH die by suicide in the next 12 months, and $2-3 \%$ over the next five years. ${ }^{3}$ The importance of adequate psychosocial assessment of persons deliberately harming themselves is therefore self evident. To assist general hospitals in improving services, the Royal College of Psychiatrists recently produced a consensus statement on standards of service for the general hospital management of adult DSH. ${ }^{4}$

The A\&E department of the Leicester Royal Infirmary has a catchment area of 0.9 million persons, and a multidisciplinary specialist service for the assessment of those presenting with DSH. This service, comprising of psychiatric nurse specialists and a social worker, is however restricted to weekdays between the hours of 9 am and $5 \mathrm{pm}$, and is dependent on $A \& E$ or ward staff to perform the initial screen and instigate referral.

Our principal objective was to examine the adequacy of the management of DSH in the $\mathrm{A} \& \mathrm{E}$ department by comparing with an "ideal" standard. ${ }^{4}$ In addition to examining the management of 934 consecutive attenders to the Leicester Royal Infirmary with a diagnosis of DSH, we also surveyed A\&E staff concerning their knowledge, confidence, attitudes, and postgraduate training in the psychosocial assessment of this behaviour.

\section{Methods}

All attendances at the Leicester Royal Infirmary A\&E department with a discharge diagnosis of "self inflicted injury" for the 12 month period April 1994 to March 1995 were retrospectively identified from the PAS (patient activity analysis) system. The year chosen for the project spanned three rotations of junior medical staff. A\&E records were located for 1592 of 1702 episodes of "self inflicted injury". From these, 658 cases were excluded as they failed to satisfy the criteria for DSH. DSH was defined as "an intentional self-injury (nonfatal), or deliberate ingestion of more than a prescribed amount of medical substances, or the deliberate ingestion of substances never intended for human consumption." The notes of the remaining 934 episodes of DSH were then examined in a standardised fashion using an instrument designed by the authors (appendix 1), based upon standards of service provision detailed by the Royal College of Psychiatrists. ${ }^{4}$ Specific consideration was given to the quality and appropriateness of assessments undertaken by nursing staff in triage, and $A \& E$ medical staff. Methods of self harm, waiting times, and final outcome (whether the patient was admitted, discharged, or if they were seen 
Table 1 Method of deliberate self harm

\begin{tabular}{lrc}
\hline Method & $n$ & $\%$ \\
\hline Paracetamol overdose & 386 & 41 \\
Benzodiazepine overdose & 160 & 17 \\
Aspirin/non-steroidal anti-inflammatory drug & & \\
$\quad$ overdose & 101 & 11 \\
Tricyclic antidepressant overdose & 75 & 8 \\
Selective serotonin reuptake inhibitor & & \\
$\quad$ overdose & 50 & 5 \\
Other antidepressant overdose & 10 & 1 \\
Other psychotropic overdose & 90 & 10 \\
Other overdose & 205 & 22 \\
Deliberate illicit substance overdose & 4 & 0.5 \\
Wrist cutting & 95 & 10 \\
Other deliberate self injury & 56 & 6 \\
Non-ingestibles & 15 & 2 \\
Carbon monoxide poisoning & 7 & 1 \\
Hanging & 6 & 0.5 \\
Other & 1 & 0 \\
\hline
\end{tabular}

Total No of episodes of DSH $=934 ; 241(26 \%)$ involved more than one method.

by a psychiatric specialist in the department) were also recorded. Attention was given, where possible, to any follow up care which was arranged before discharge from A\&E. Simple demographic details including sex, age, residential status, marital status, and source of referral were also noted.

A brief questionnaire was circulated to current $A \& E$ medical and nursing staff inquiring into four areas: knowledge, confidence, attitudes, and postgraduate training in the psychosocial assessment of DSH (appendix 2). Data were analysed using SPSS for Windows, ${ }^{6}$ demographic details are expressed as means (SD), and categorical data compared using the $\chi^{2}$ test with degrees of freedom (df).

\section{Results}

ACCIDENT AND EMERGENCY MANAGEMENT OF DELIBERATE SELF HARM

There were 934 episodes of DSH involving 854 patients of mean age of 32 (SD 14.2) years, range 16 to 90 . The mean age for women (30.5 (12.7) years) was significantly lower (two tailed $t$ test, $\mathrm{P}=0.002$ ) than for men (33.4 (15.3) years). There was a relatively even sex distribution, with 453 men (48\%), and 481 (52\%) women. Marital status was recorded for 644 persons: 296 (50\%) were single, 208 (35\%) married or cohabiting, and only 92 (15\%) separated, divorced, or widowed. By far the majority of attendances were initiated by the public or patient $(88 \%)$, and only $5 \%$ by general practitioners.

Overdose was by far the most common method of DSH, and was involved in 855 cases $(91.5 \%)$. Table 1 illustrates the method of DSH for the episodes: $241(26 \%)$ involved more than method, and the majority of these were overdoses of more than one substance. Evidence of recent alcohol consumption was recorded for only 378 episodes (40\%).

Time of attendance was recorded in 934 cases, and was categorised into three groups: $398(43 \%)$ were in the evening (between the hours of $5 \mathrm{pm}$ and midnight); 285 (30\%) during normal working hours ( 9 am to $5 \mathrm{pm}$ ); and $251(27 \%)$ during the night (12 midnight to 9 am). In this study, 772 persons ( $83 \%$ ) were assessed by a triage nurse on arrival at $A \& E$; a record of degree of clinical urgency was found
Table 2 AËE doctor's psychosocial assessment of deliberate self harm (DSH)

\begin{tabular}{lcc}
\hline & \multicolumn{2}{c}{ Overall $(n=931)$} \\
\cline { 2 - 3 } Criteria & $n$ & $\%$ \\
\hline Conscious level & 456 & 49 \\
Psychiatric history & 621 & 67 \\
Medical history & 469 & 50 \\
Mental state examination & 472 & 51 \\
Recent stresses & 515 & 55 \\
Previous DSH & 442 & 47 \\
Employment status & 90 & 10 \\
Living conditions & 405 & 44 \\
Threats of DSH & 219 & 23 \\
Alcohol dependence & 100 & 11 \\
Illicit drug use & 63 & 7 \\
Suicide intent & 649 & 70 \\
Risk of further DSH & 432 & 46 \\
\hline
\end{tabular}

in $407(53 \%)$, distress in $144(19 \%)$, and likelihood of waiting for treatment in $15(2 \%)$. The legibility of A\&E medical staffs' entries was very good, with 892 (96\%) coded as legible, and only $38(4 \%)$ as partially legible.

The adequacy of the A\&E doctors' psychosocial assessment is considered in detail in table 2. Outcome was recorded in a variety of ways, and detailed outcome is shown in table 3 . Of the 241 patients seen only by the A\&E doctor and discharged without psychiatric follow up, there was a clear indication that the general practitioner had been contacted in 31 cases, 29 were advised to contact the DSH team in the future, 12 were discharged into police custody, and one was referred to social services.

We have further simplified outcome into three categories reflecting clinical responsibility for immediate management decisions. These are:

(1) Assessed only by A\&E medical staff, and discharged directly home $(n=291)$;

(2) Referred for specialist assessment in $A \& E$ ( $n=210$ );

(3) Admitted to medical or surgical wards ( $n=$ 423).

The adequacy of the psychosocial information recorded by the $A \& E$ doctor for these outcomes is outlined in table 4 , and as expected the frequency of information recorded varied significantly between outcomes. In addition, mental state $\left(\chi^{2}=6.6, \mathrm{df}=1, \mathrm{P}=\right.$ $0.01)$, history of threats $\left(\chi^{2}=6, \mathrm{df}=1, \mathrm{P}=\right.$ $0.014)$, suicide intent $\left(\chi^{2}=6.9, \mathrm{df}=1, \mathrm{P}=\right.$ $0.009)$, and suicide risk $\left(\chi^{2}=8.95, \mathrm{df}=1, \mathrm{P}=\right.$ 0.003 ) were more likely to be recorded if the patient was referred for specialist assessment than discharged directly from the department.

Table 3 Deliberate self harm (DSH) attendances at $A \mathcal{E} E$ : main outcome

\begin{tabular}{lcc}
\hline Outcome & $n(928)$ & $\%$ \\
\hline $\begin{array}{l}\text { Left department before assessed by A\&E } \\
\text { doctor }\end{array}$ & 4 & 0.5 \\
$\begin{array}{l}\text { Discharged home by A\&E doctor with no } \\
\text { psychiatric follow up }\end{array}$ & 241 & 26 \\
$\begin{array}{l}\text { Referred for specialist assessment in A\&E, but } \\
\text { left before seen }\end{array}$ & 46 & 5 \\
$\begin{array}{l}\text { Discharged by A\&E doctor with psychiatric } \\
\text { outpatient appointment }\end{array}$ & 50 & 5 \\
$\begin{array}{l}\text { Discharged by specialist with psychiatric } \\
\text { outpatient appointment }\end{array}$ & 64 & 7 \\
$\begin{array}{l}\text { Admitted to psychiatry ward } \\
\text { Discharged by specialist with no follow up }\end{array}$ & 55 & 6 \\
Admitted to medical/surgical ward & 423 & 5 \\
\hline
\end{tabular}




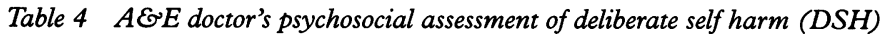

\begin{tabular}{llllll}
\hline & & \multicolumn{2}{l}{ Outcome $(n=924)$} & \\
\cline { 2 - 5 } Criteria & & $\begin{array}{l}\text { Discharged by } \\
\text { AEE Dr }\end{array}$ & $\begin{array}{l}\text { Referred to } \\
\text { specialist }\end{array}$ & $\begin{array}{l}\text { Medical } \\
\text { admission }\end{array}$ & $\begin{array}{l}\text { SignificanceP } \\
\text { value }\left(\chi^{2}\right)\end{array}$ \\
\hline Conscious level & Yes & 119 & 92 & 244 & $<0.001$ \\
& No & 172 & 118 & 179 & \\
Psychiatric history & Yes & 174 & 143 & 302 & 0.005 \\
& No & 117 & 67 & 121 & \\
Medical history & Yes & 132 & 89 & 246 & $<0.001$ \\
Mental state & No & 159 & 121 & 177 & \\
examination & & & & & \\
& Yes & 148 & 131 & 187 & $<0.001$ \\
Recent stresses & No & 187 & 79 & 236 & \\
& Yes & 166 & 129 & 217 & 0.043 \\
Previous DSH & No & 125 & 81 & 206 & \\
& Yes & 134 & 112 & 193 & 0.15 \\
Employment status & No & 157 & 98 & 230 & \\
& Yes & 33 & 27 & 29 & 0.03 \\
Living conditions & No & 258 & 183 & 394 & \\
& Yes & 131 & 108 & 163 & 0.007 \\
Threats of DSH & No & 160 & 102 & 260 & \\
& Yes & 62 & 65 & 91 & 0.02 \\
Alcohol dependence & No & 229 & 145 & 332 & \\
Yes & 25 & 26 & 49 & 0.32 \\
Illicit drug use & No & 266 & 184 & 374 & \\
& Yes & 17 & 15 & 31 & 0.72 \\
Suicide intent & No & 274 & 195 & 392 & \\
& Yes & 217 & 177 & 249 & $<0.001$ \\
Risk of further DSH & No & 74 & 33 & 174 & \\
& Yes & 148 & 135 & 144 & $<0.001$ \\
& No & 143 & 75 & 279 & \\
\hline
\end{tabular}

Table 5 Feelings evoked among $A \mathcal{E} E$ Estaff when dealing with cases of deliberate self harm

\begin{tabular}{|c|c|c|c|c|c|c|}
\hline \multirow[b]{2}{*}{ Feeling } & \multicolumn{2}{|c|}{ Overall sample $(n=54)$} & \multicolumn{2}{|c|}{ Medical staff } & \multicolumn{2}{|c|}{ Nursing staff } \\
\hline & $n$ & $\%$ & $n$ & $\%$ & $n$ & $\%$ \\
\hline Indifference & 19 & 35 & 11 & 61 & 8 & 22 \\
\hline Sympathy & 36 & 66 & 7 & 39 & 29 & 80 \\
\hline Anger & 11 & 21 & 2 & 11 & 9 & 26 \\
\hline Helplessness & 23 & 43 & 2 & 11 & 21 & 60 \\
\hline Anxious & 9 & 17 & 0 & 0 & 9 & 26 \\
\hline
\end{tabular}

There were significant differences when comparing simple outcome by time of admission $\left(\chi^{2}=17.8, \mathrm{df}=4, \mathrm{P}=0.001\right)$; in particular $\mathrm{A} \& \mathrm{E}$ medical staff were far more likely to discharge a patient home themselves at night than to refer for specialist assessment $\left(\chi^{2}=\right.$ 16.2, $\mathrm{df}=2, \mathrm{P}=0.0003$ ).

Patients aged 55 or older were significantly more likely to be admitted to a medical ward $\left(\chi^{2}=34, \mathrm{P}<0.001\right)$. When considering the cases of those aged 65 years or more $(n=42)$, 31 were admitted medically, four were admitted to psychiatry wards, a further three were assessed by specialists and psychiatric outpatient follow up arranged, and only four (9.5\%) were discharged directly by $A \& E$ medical staff without follow up.

\section{STAFF QUESTIONNAIRE}

There was an overall response rate of $59 \%$ for the staff questionnaire, with replies from 18 of 28 medical staff, and 36 of 63 trained nursing staff. Only 13 staff members (24\%) had received postqualification training in the psychosocial assessment of DSH, and this usually took the form of a short lecture, or seminar. Overall $33(61 \%)$ of respondents admitted to having regular doubts about their ability and confidence in the assessment of cases of DSH, and $17(32 \%)$ had occasional doubts. Respondents clearly felt further training would be beneficial, in particular in the areas of suicide intent $(65 \%, \mathrm{n}=35)$, mental state examination $(83 \%, n=45)$, the use of the Mental Health Act and Common Law $(85 \%, n=46)$, and the organisation of and access to mental health services $(76 \%, n=41)$. Most $(93 \%, n=50)$ A\&E staff felt they would appreciate supervision by a mental health professional, but the overall sentiment was in favour of a 24 hour on site specialist service. The feelings experienced by $\mathrm{A} \& \mathrm{E}$ staff when dealing with cases of $\mathrm{DSH}$ are included in table 5 .

\section{Discussion}

The close links between suicide and DSH emphasise the importance of an adequate psychosocial assessment for all persons who deliberately harm themselves. ${ }^{7}$ We based our audit upon the Royal College of Psychiatrists' consensus statement on standards for service provision for the general hospital management of adult DSH, as this considers in detail the assessment performed by non-medical staff in the A\&E department. ${ }^{4}$

There were some very important differences from previous studies. Most striking was the almost equal numbers of both sexes; previous studies have female to male ratios of $1.5: 1,{ }^{89}$ though there has been a trend towards a narrowing of the sex difference. ${ }^{1}$ In addition, only $46 \%$ of cases were admitted to medical wards, and $6 \%$ to psychiatry wards, substantially less than the admission rate of $70 \%$ found in Nottingham in $1986,{ }^{8}$ and more recently from Bristol in $1993 .{ }^{10}$ With over half the sample not being admitted directly to acute medical or surgical wards the responsibility for an adequate risk assessment therefore lies with $\mathrm{A} \& \mathrm{E}$ medical staff. It may be that increasing pressure on acute hospital beds has resulted in changes of admission policy, with fewer patients admitted for psychosocial reasons.

Patients in A\&E include some who are in urgent physical danger, and some who may leave hospital precipitately because of an abnormal mental state. Nurse triage should therefore identify whether the person is physically fit to wait, or in distress, and whether they are likely to wait to be seen by the $A \& E$ doctor.

In the psychosocial assessment of self harm it is important that information is collected in a systematic way and carefully recorded. Most appropriate risk factors were recorded in approximately half the sample though in particular key areas, such as suicide intent and psychiatric history, the rate was much higher. There was also evidence of selective documentation, with those referred for specialist assessment significantly more likely to have mental state, suicide intent and risk, and history of threats recorded than those discharged from the department. In addition, conscious level and medical history were documented more often in those admitted. This selective recording of risk factors might suggest that in many instances appropriate questions were asked, but not always documented. In addition, in some cases important information concerning risk may have been recorded at previous attendances. There were, however, disappoint- 
ingly low rates of recording information concerning alcohol and substance misuse, especially as alcohol use is associated with repetition, ${ }^{11}$ and substance misuse with suicide in young people. ${ }^{12}$

Deliberate self harm in the elderly closely resembles successful suicide, and elderly persons who harm themselves often have a high degree of intent. ${ }^{13}$ It is therefore reassuring that the vast majority of those over 65 years of age were admitted, and very few were discharged without specialist assessment or follow up. Of considerable concern was that in 46 cases of referral for specialist assessment $(5 \%$ of attendances), the patient left the department before being seen. The fact that a referral had been instigated implied that they were considered to be at risk. During normal office hours a non-medical member of the DSH team is based within the hospital, but after hours specialist assessment is provided by a nonresident registrar grade psychiatrist; this may also account for the significantly fewer cases referred for specialist assessment at night.

Perhaps of most significance was the $A \& E$ medical and nursing staffs' own perception of their knowledge and confidence, and their clearly stated desire for further training. There has been debate as to whether negative attitudes towards persons who harm themselves affect the quality of assessment they receive. Black and Creed $^{14}$ found this to be unlikely, and in this particular study, we found that although indifference was frequently experienced, anger was rare.

There is little to suggest that the situation in Leicester is significantly different from that in the majority of UK cities. Leicester is fortunate in that there is an established multidisciplinary DSH team, and a specific on call rota to provide psychiatric assessment. A national survey of practice in the late $1980 \mathrm{~s}^{15}$ suggested that there had been little movement towards the use of specific self harm teams and multidisciplinary working suggested in DHSS guidelines. The study, however, reveals a clear need for improved planning and delivery of services. A preprinted checklist or stamp for both the nurse triage, and for the A\&E doctors' psychosocial and risk assessment, might ensure more thorough inquiry and documentation. Although risk factors have low predictive value for subsequent suicide, they are useful in prompting adequate inquiry and as a check on clinical assessments. The Health Advisory Service review on suicide prevention emphasises the importance of adequate psychosocial assessment of $\mathrm{DSH}$ patients in $\mathrm{A} \& \mathrm{E}$, and includes a useful checklist for the psychiatric assessment. ${ }^{7}$ A\&E staff require suitable training in the assessment of DSH, which needs to be included in their induction week, with more specific information concerning local policies and psychiatric services. For those discharged from $A \& E$, written information about follow up arrangements needs to be provided. For others with no specific appointments, or who are reluctant to wait to see a specialist but are not detainable under the Mental Health Act, it may be worthwhile considering a card with the phone numbers of local DSH or psychiatry services, as piloted in Bristol. ${ }^{16}$ Some centres advocate the use of short stay admission facilities for those requiring time to recover from the neuropsychiatric effects of self poisoning, or in cases where time is needed to ensure adequate assessment and care packages. ${ }^{9}$

Local specialist services need to be actively involved in local planning groups, and assist in providing the appropriate postqualification training for $A \& E$ staff. Extending the $\mathrm{DSH}$ team service to the evenings, when a considerable proportion of these patients attend $A \& E$, or the provision of a resident on call psychiatry service may facilitate referral and improve liaison.

In the wake of this particular study, certain service improvements have been implemented in Leicester, in particular the use of preprinted checklists for risk assessment, and extension of the working hours of the DSH team. Whether service improvements lead to better outcome in terms of prevention of suicide and repetition of DSH requires further evaluation.

We thank Leicestershire Health for providing us with financia wert, and the staff of the Leicester Royal Infirmary accident and emergency department for completing the questionnaires.

\section{Appendix 1}

Deliberate self harm audit: A\&E department, Leicester Royal Infirmary

INFORMATION COLLECTED

$(\mathrm{NK}=$ not known; NA $=$ not applicable $; \mathrm{NFA}=$ no fixed abode)

Audit code No: $\quad$ A\&E No:

Date of attendance:

Surname:

Forename(s):

Age:

Date of birth:

Male/Female

Single/Separated or divorced/Married or cohabiting/ Widowed/NK

Permanent address/NFA/NK

Source of referral: GP/Police/999/Psychiatric unit/ Other hospital/Self/NK

Number of previous attendances in last 12 months:

Method of DSH (give details, eg, drugs taken, dosage, quantity, etc)

Alcohol associated with DSH? Yes/No/Missing/NK Time of admission to A\&E

Delay to see A\&E doctor

Delay to see psychiatric specialist

Time: $\frac{N A}{(\mathrm{~h} / \mathrm{min})}$

\section{ASSESSMENT BY A\&E NURSE Yes/No/NA}

If yes, does assessment include:

Physical fitness to wait for doctor? Yes/No/NA/NK Presence of obvious distress? Yes/No/NA/NK Likelihood that patient will wait to see doctor? $\mathrm{Yes} / \mathrm{No} / \mathrm{NA} / \mathrm{NK}$

Legibility of assessment: Legible/Partly legible/ Illegible

ASSESSMENT BY A\&E DOCTOR Yes/No/NA/

Patient refusing/Missing or NK

Grade of doctor: SHO/Registrar/Senior registrar/ Consultant/Other/NA/NK

If $\mathrm{SHO}$, was patient discussed with other grade of A\&E doctor? Yes/No/NA/NK Conscious level: Alert/Mildly drowsy/Very drowsy/ Unconscious NA/NK

Legibility of assessment: Legible/Partly legible/ Illegible

Does assessment include:

Code individually as appropriate: $\quad$ Yes/No/NA/

Conscious level Patient refusing/missing or NK)

If patient not fully alert was assessment deferred? 
Psychiatric history

Medical history

Mental state examination

Recent stresses

Previous DSH

Employment etatus

Living conditions

Threats of DSH or note

Chronic alcohol use

Illicit drug use

Assessment of suicide intent

Risk of further self harm

Arrangements for psychiatric follow up

OUTCOME

Was patient given any written information about how to get help?

Yes/No/NK/NA

Appendix 2

Deliberate self harm audit: A\&E Department,

Leicester Royal Infirmary

PERSONNEL QUESTIONNAIRE

1. Since qualifying (ie, medical degree, RGN, etc) have you received training in the psychosocial assessment of cases of deliberate self-harm? Yes/No

2. If yes, which of the following best describes the training you received? Short lecture or seminar/ Half day course/Other, please specify:

3. Who provided the training? Psychiatrist/A\&E nursing staff/DSH team/Nursing training school or tutor/A\&E medical staff/External agency

4. Have you ever had full time postqualification experience of working in psychiatry? Yes/No

5. Do you ever doubt your ability in the assessment of DSH cases? Never/Rarely/ Frequently/Always

6. Do you ever doubt your ability in the following areas? (You may tick more than one) Seriousness of the attempt/Mental state examination/Medico-legal issues/Arrangements for follow up

7. Please indicate the areas in which you feel you would benefit from further training: Assessment of seriousness of the attempt Assessment of mental state

Use of Mental Health and Common Law Communication and interview skills Organisation and access to mental health services

8. Would you welcome supervision by a mental health specialist in the management of DSH patients? Yes/No

9. How do you feel towards DSH patients (you may tick more than one): Indifference/Sympathy/Anger/ Helpless/Anxious
10. How adequate do you consider the services provided by:

DSH team: Excellent/Very good/Barely adequate/ Poor

Out of hours on-call psychiatrists: Excellent/Very good/Barely adequate/Poor

Thank you for taking time to complete this questionnaire. All information will be treated in the strictest confidence. Please return the completed questionnaire in the envelope provided to: Marie Beach, Psychiatry for the Elderly, Leicester General Hospital.

1 Hawton K, Fagg J. Trends in deliberate self-poisoning and self injury in Oxford, 1976-90. BMJ 1992;304:1409-11.

2 Dennis M, Owens D, Jones S. Epidemiology of deliberate self-poisoning - trends in hospital attendances. Health Trends 1990;3:125-6.

3 Hawton K, Fagg J. Suicide and other causes of death, following attempted suicide. $\mathrm{Br} J$ Psychiatry 1988;152: 359-66.

4 The General Hospital Management of Adult Deliberate Self-Harm. A consensus statement on standards for service provision. Council Report CR32. London: The Royal College of Psychiatrists, 1994.

5 Morgan HG. Death wishes? The understanding and management of deliberate self-harm. Chichester: John Wiley, 1979.

6 SPSS for Windows. Statistical package for social sciences. Chicago: SPSS, 1993.

7 Williams R, Morgan HG. Suicide prevention: the challenge confronted. A manual of guidance for the purchasers and providers of mental health care. London: HMSO, 1994.

8 Owens $D$, Dennis $M$, Jones S, Dove A, Dave S. Self-poisoning patients discharged from Accident and Emergency: risk factors and outcome. J R Coll Physicians Lond 1991;25:218-22.

9 Platt S, Hawton K, Krietman N, Fagg J, Foster J. Recent clinical and epidemiological trends in parasuicide in Edinburgh and Oxford: a tale of two cities. Psychol Med 1988; 18:405-18.

10 Evans J, Johnson C, Stanton R, Morgan HG. How to establish case registers: II. Non-fatal deliberate self-harm. Psychiatr Bull 1996;20:403-5.

11 Buglass D, Horton J. The repetition of parasuicide: a comparison of three cohorts. Br J Psychiatry 1974;125:168-74.

12 Hawton K, Fagg J, Platt S, Hawkins M. Factors associated with suicide after parasuicide in young people. BMJ 1993; 306:1641-4.

13 Dennis M, Lindesay J. Suicide in the elderly, the United Kingdom perspective. Int Psychogeriatr 1995;7:263-74.

14 Black D, Creed F. Assessment of self-poisoning by psychiatrists and junior medical staff. J R Soc Med 1988; 81:97-9.

15 Butterworth E, O'Grady T. Trends in the assessment of cases of deliberate self-harm. Health Trends 1989;21:61.

16 Morgan H, Jones E, Owen J. 1993. Secondary prevention of non-fatal deliberate self-harm. The Green Card Study. Br J Psychiatry 1993;163:111-13. 